\title{
Signal-to-Idler Conversion Penalty in AIGaAs-on-Insulator Wavelength Converter
}

Kaminski, Pawel Marcin; Da Ros, Francesco; Porto da Silva, Edson; Pu, Minhao; Yankov, Metodi Plamenov; Semenova, Elizaveta; Yvind, Kresten; Oxenløwe, Leif Katsuo; Forchhammer, Søren; Galili, Michael

\section{Published in:}

CLEO: Science and Innovations 2018

Link to article, DOI:

10.1364/CLEO_SI.2018.STU4C.6

Publication date:

2018

Document Version

Peer reviewed version

Link back to DTU Orbit

Citation (APA):

Kaminski, P. M., Da Ros, F., Porto da Silva, E., Pu, M., Yankov, M. P., Semenova, E., Yvind, K., Oxenløwe, L. K., Forchhammer, S., \& Galili, M. (2018). Signal-to-Idler Conversion Penalty in AlGaAs-on-Insulator Wavelength Converter. In CLEO: Science and Innovations 2018 [Paper STu4C.6] Optical Society of America. https://doi.org/10.1364/CLEO_SI.2018.STu4C.6

\section{General rights}

Copyright and moral rights for the publications made accessible in the public portal are retained by the authors and/or other copyright owners and it is a condition of accessing publications that users recognise and abide by the legal requirements associated with these rights.

- Users may download and print one copy of any publication from the public portal for the purpose of private study or research.

- You may not further distribute the material or use it for any profit-making activity or commercial gain

- You may freely distribute the URL identifying the publication in the public portal 


\title{
Signal-to-Idler Conversion Penalty in AlGaAs-on-Insulator Wavelength Converter
}

\author{
P. M. Kaminski, F. Da Ros, E. P. da Silva, M. Pu, M. P. Yankov, E. Semenova, K. Yvind, \\ L. K. Oxenløwe, S. Forchhammer, and M. Galili \\ DTU Fotonik, Technical University of Denmark, DK-2800 Kgs. Lyngby, Denmark, pkam@fotonik.dtu.dk
}

\begin{abstract}
A wavelength converter based on AlGaAsOI waveguide is characterized by varying the input signal quality. Signal-to-idler conversion penalty is measured in terms of effective received SNR, and trade-offs between penalty and converted signal quality are outlined.

OCIS codes: (190.4380) Nonlinear optics, four-wave mixing, (060.0060) Fiber optics and optical communications,
\end{abstract}

\section{Introduction}

With the ever-increasing traffic demands, telecommunication systems are currently facing a number of challenges to meet future requirements, while maintaining cost-efficiency and reliability. All-optical networks call for effective wavelength conversion schemes to ease traffic grooming and resolve wavelength contention [1]. Scenarios based on four-wave mixing (FWM) in $\chi^{(3)}$ media have drawn much attention as they may exhibit lower power consumption and latency, operate over broad bandwidth, while also supporting transparency to modulation formats and data rates [24]. Integrated FWM platforms are currently becoming promising candidates to be employed as nonlinear media for the process because of high nonlinearity and small footprint [2-6]. In particular, AlGaAs-on-insulator (AlGaAsOI) offers low propagation losses, strong nonlinearity and large bandgap for two-photon absorption free operation at $1550 \mathrm{~nm}$ [4-6]. The interest in compact wavelength converters is further driven by the several potential applications of wavelength conversion. Given a proper link design, the generated wavelength-shifted idler also offers a possibility of all-optical Kerr nonlinearity compensation by means of optical-phase conjugation. In either case, the wavelength converting / phase conjugating element is located within the network, and will most often have to process degraded signals far from transmitter and receiver. Wavelength converters have been mainly characterized by looking at the conversion penalty for input signals with maximum optical signal-to-noise ratio (OSNR) and performing noise loading at the receiver side [3-4,7]. The impact of input signal OSNR and pump quality has been preliminarily analyzed in $[8,9]$ and in $[7,9]$ optical noise figures have been estimated from input-to-output OSNR differences.

In this work, we extend previous investigations by focusing on an experimental characterization of the performance of an AlGaAsOI-based wavelength converter with respect to the signal OSNR and power at the input to the nonlinear waveguide. We go beyond relying on the OSNR as a quality metric and demonstrate the relation between input signalto-noise ratio (SNR) and output idler SNR, from which a conversion penalty of the wavelength converter under test can be derived. The analysis is conducted for five wavelength-division multiplexed (WDM) 16-quadrature amplitude modulation (16-QAM) channels at 16-GBd.

\section{Experimental setup}

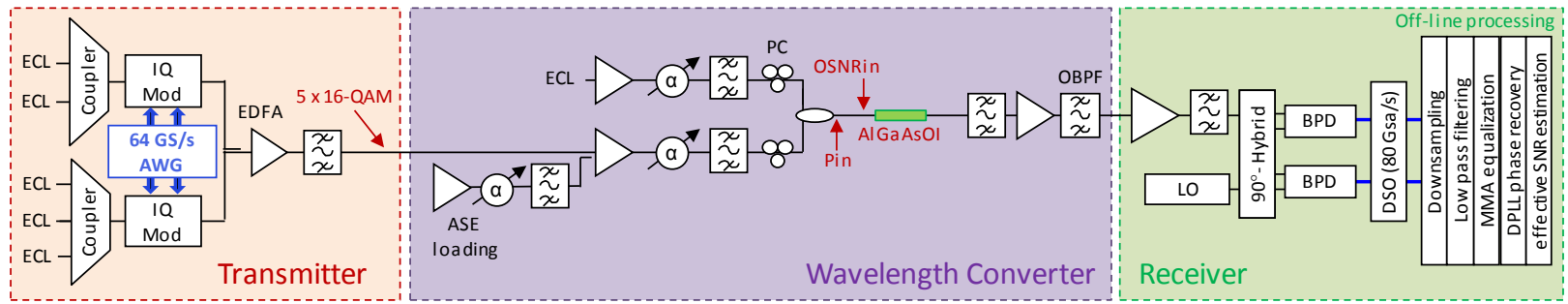

Fig. 1. Experimental setup employed for wavelength converter characterization.

The experimental setup is given in Fig. 1. At the transmitter side, five WDM channels on a $25-\mathrm{GHz}$ grid are generated from external cavity lasers (ECLs), with 16-QAM test data at 16-GBd subsequently modulated onto the corresponding waves with two external IQ modulators (one for even and one for odd channels). All channels are coupled together, amplified with an erbium-doped fiber amplifier (EDFA) and launched directly into a wavelength converter based on FWM in a $9 \mathrm{~mm}$ AlGaAsOI waveguide. The setup operates under single pump-configuration. The signal is combined with a continuous-wave pump from a sub-kHz linewidth fiber laser. The pump is set at $1550 \mathrm{~nm}$ with $20 \mathrm{dBm}$ of pump power at the waveguide input. The nonlinear waveguide is characterized by an insertion loss of $6.8 \mathrm{~dB}$, an output conversion efficiency $(\mathrm{CE})$ of $-18 \mathrm{~dB}$, and a broad conversion bandwidth which extends beyond Cband, as shown in Fig 2(a). The signal OSNR at the input to the converter (OSNRin) is controlled by adding amplified 
spontaneous emission (ASE) noise from an extra EDFA, and the total input signal power (Pin) is varied with a variable optical attenuator (VOA) in the signal path. Polarization controllers (PC) for both pump and signal ensure alignment of the waves to the waveguide TE-mode for minimum loss and maximum FWM. At the output of the converter, the generated idler is selected with proper filtering, amplified and received with a coherent receiver followed by offline digital signal processing, including equalization, carrier phase recovery and SNR estimation. The effective SNR is estimated from the transmitted QAM symbols $x_{k}$ and their corresponding received samples $y_{k}$ after all DSP blocks at times $k=[1 ; K]$ as $S N R_{\text {eff }}=\mathbb{E}_{k}\left[\left|x_{k}\right|^{2}\right] / \mathbb{E}_{k}\left[\left|y_{k}-x_{k}\right|^{2}\right]$ as in [10].

\section{Conversion results}
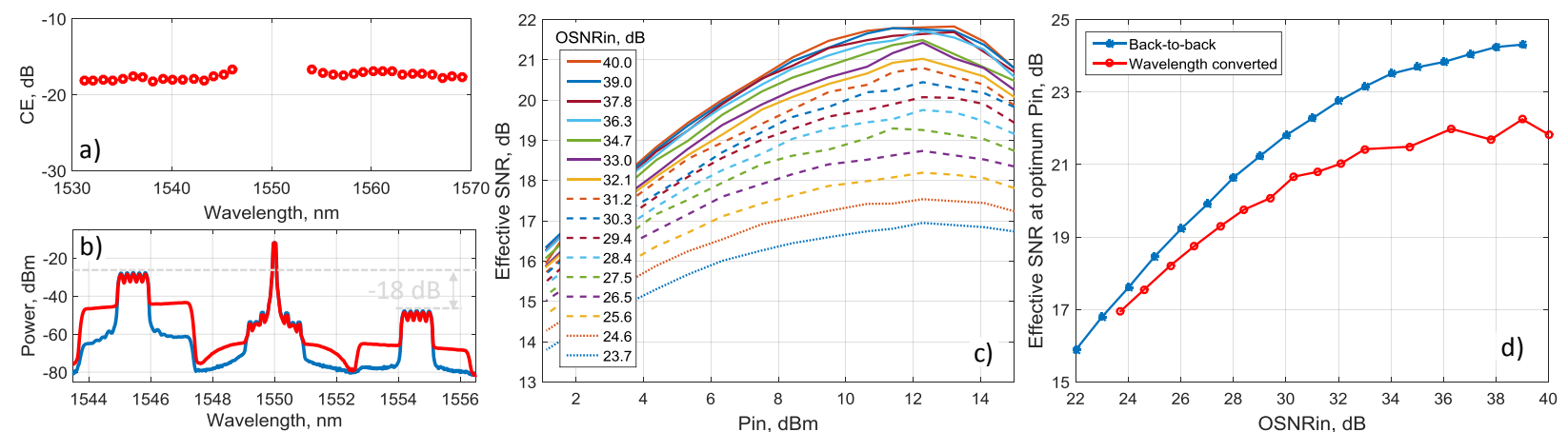

(c) Measured SNR in terms of varying input signal OSNRin and input Pin, and (d) SNR as a function of OSNRin at optimum Pin compared to back-to-back.

The optical spectrum at the output of the waveguide is shown in Fig. 2(b) for the maximum (blue, idler OSNR $>30$ $\mathrm{dB}$ ) and minimum (red, idler OSNR $\approx 23 \mathrm{~dB}$ ) input signal OSNR at optimum Pin. The performance of the wavelengthconverted idler has been evaluated in terms of effective idler SNR as a function of the input signal OSNRin and signal power Pin. The results are shown in Fig. 2(c). The SNR increases with OSNRin, and the optimum Pin for conversion remains constant at approx. $12 \mathrm{dBm}$. Fixing Pin to its optimum value, the idler SNR is plotted as a function of OSNRin in Fig. 2(d), and the results are benchmarked against a back-to-back reference measured at the waveguide input (pump OFF). At low OSNRin, the input signal OSNR provides the dominant noise contribution determining the idler performance and resulting in SNR penalty below $0.5 \mathrm{~dB}$. At high OSNRin, the idler OSNR is limited by the CE and ASE noise leakage from the pump (see Fig. 2(b)) and the received idler SNR is degraded by up to $2 \mathrm{~dB}$. The relation between the curves provides more insight into the signal-to-idler conversion penalties, and outlines an important trade-off between the SNR penalty and the performance of the generated idler. Higher CE (pump power) may be beneficial for processing high OSNRin signals with lower penalty, while the current CE is sufficient for low OSNRin operation.

\section{Conclusions}

This work has experimentally demonstrated the impact of input signal quality on the performance of all-optical wavelength conversion based on FWM in AlGaAsOI waveguide. The study shows that optimum signal input power is independent of input signal OSNR. However, signal-to-idler conversion penalty defined in terms of effective SNR is strongly dependent on the input signal OSNR, for a given CE, with the penalty varying from $0.5 \mathrm{~dB}$ to $2 \mathrm{~dB}$. The connection between the input OSNR and output SNR establishes a trade-off between achievable penalty, and the overall performance of the wavelength-converted signal.

\section{Acknowledgements}

This work was supported by the DNRF Research CoE, SPOC (ref. DNRF123), and the DFF project NANO-SPECs (DFF-4005-00558B).

\section{References}

[1] M. Saleh et al., "All-optical networking evolution benefits challenges and future vision", Proc. IEEE, 100(5), 1105 (2012).

[2] C. Lacava et al., "Nonlinear silicon photonic signal processing devices for future optical networks", Applied Sciences, 7(1), 103 (2017).

[3] I. Sackey et al., "1.024 Tb/s wavelength conversion in a silicon waveguide with reverse-biased p-i-n junction", Opt. Expr. 25(18), 21229 (2017).

[4] F. Da Ros et al., "Characterization and optimization of a high-efficiency AlGaAs-On-Insulator-based wavelength converter for 64- and 256QAM signals", JLT 35(17), 3750 (2017).

[5] M. Pu et al., "Efficient frequency comb generation in AlGaAs-on-insulator", 3(8), 823 (2016).

[6] L. Ottaviano et al., "Low-loss high-confinement waveguides and microring resonators in AlGaAs-on-insulator", Opt. Lett. 41(17), 3996 (2016).

[7] H. Nguyen Tan et al., "On the cascadability of all-optical wavelength converter for high-order QAM formats", JLT 34(13), 3194 (2016).

[8] H. Kishikawa et al., "All-optical wavelength preserved modulation format conversion from PDM-QPSK to PDM-BPSK using FWM and interference", JLT 34(23), 5505 (2016).

[9] R. Elschner, "Parametric amplification and wavelength conversion of phase-modulated signals", Doctoral thesis (2011).

[10] M.P. Yankov et al., "Constellation shaping for WDM systems using 256QAM/1024QAM with probabilistic optimization", JLT 34(22), 5146 (2016). 